\title{
A recursive reduction of tensor Feynman integrals
}

\author{
Th. Diakonidis ${ }^{\text {a }}$, J. Fleischer ${ }^{\text {b }}$, T. Riemann ${ }^{\text {a,* }}$, J.B. Tausk ${ }^{\text {a }}$ \\ a Deutsches Elektronen-Synchrotron, DESY, Platanenallee 6, 15738 Zeuthen, Germany \\ ${ }^{\mathrm{b}}$ Fakultät für Physik, Universität Bielefeld, Universitätsstr. 25, 33615 Bielefeld, Germany
}

\section{A R T I C L E I N F O}

\section{Article history:}

Received 24 July 2009

Received in revised form 20 November 2009

Accepted 20 November 2009

Available online 28 November 2009

Editor: A. Ringwald

\section{Keywords:}

NLO computations

QCD

QED

Feynman integrals

\begin{abstract}
A B S T R A C T
We perform a new, recursive reduction of one-loop $n$-point rank $R$ tensor Feynman integrals [in short: $(n, R)$-integrals] for $n \leqslant 6$ with $R \leqslant n$ by representing $(n, R)$-integrals in terms of $(n, R-1)$ - and $(n-1, R-1)$-integrals. We use the known representation of tensor integrals in terms of scalar integrals in higher dimension, which are then reduced by recurrence relations to integrals in generic dimension. With a systematic application of metric tensor representations in terms of chords, and by decomposing and recombining these representations, a recursive reduction for the tensors is found. The procedure represents a compact, sequential algorithm for numerical evaluations of tensor Feynman integrals appearing in next-to-leading order contributions to massless and massive three- and four-particle production at LHC and ILC, as well as at meson factories.
\end{abstract}

(c) 2009 Elsevier B.V. All rights reserved.

\section{Introduction}

For the evaluation of next-to-leading order contributions to processes at high energy colliders like LHC and ILC, but also at meson factories, one needs an efficient and reliable treatment of $(n, R)$-integrals, i.e. $n$-point Feynman integrals with tensor rank $R$. Typically $n \leqslant 6$ and $R \leqslant n$ may be needed for final states with massive particles. For $n \leqslant 4$ the Passarino-Veltman reduction [1] may be applied. For $n=5,6$ there are a variety of reduction schemes; for an overview, see e.g. [2-4]. In a recent article [5], we derived such a tensor reduction scheme for pentagons and hexagons using the Davydychev-Tarasov approach [6,7] for tensors of rank $R \leqslant 3$. Recurrence relations to reduce dimensions and indices have been applied with systematic use of signed minors [8] as described in [9]. Simplifications were derived in [5] for $n=5, R=2,3$ and $n=6, R=2,3,4$. A corresponding numerical Mathematica package hexagon.m is publicly available at http://prac.us.edu.pl/ gluza/hexagon/ [10].

In this Letter, we present a new, recursive tensor reduction which extends the reduction up to $(6,6)$ - and $(5,5)$-integrals. In this reduction tensor integrals with $n \leqslant 4$ occur which can also be dealt with in a recursive manner. This will be sufficient to evaluate the one-loop amplitudes of four-particle production at LHC and ILC. The new reductions rest on a new master formula, Eq. (16)

\footnotetext{
* Corresponding author.

E-mail addresses: Theodoros.Diakonidis@desy.de (Th. Diakonidis), Fleischer@physik.uni-bielefeld.de (J. Fleischer), Tord.Riemann@desy.de (T. Riemann), Bas.Tausk@desy.de (J.B. Tausk).
}

for five-point functions and corresponding ones for simpler functions. In principle, the tensor integrals with $n \leqslant 4$ might be treated following [1], e.g. with the Fortran package LoopTools/FF [11,12]. LoopTools treats loops with massive propagators for $n \leqslant 5,{ }^{1}$ and Golem [13] with massless propagators for $n \leqslant 6$. Unfortunately, there is no publicly available numerical Fortran package with a stable treatment of both massive and massless particles in the loop. In this situation, it appears natural to work out the complete reduction scheme for the whole chain of tensors in a systematic way.

The tensor reductions given for $n \leqslant 4$ in [1] express the tensors in terms of scalar 1- to 4-point functions. For tensor 5-point functions, reductions to tensor 4-point functions with rank less by one have been presented in [14] and [15,16], and also in [17,18] tensor recursions are discussed. A representation of scalar $(N+1)$-point functions (including integrals with powers of the loop momentum in the numerator) in terms of $N$-point functions in $N$ integer dimensions was derived in [19]. For $N=4$, such a representation was derived already in [8]. The general case of tensor integrals using dimensional regularization was treated in a series of papers [20-23], thereby allowing also for massless particles.

Our reductions express $(n, R)$-integrals recursively in terms of $(n, R-1)$ - and $(n-1, R-1)$-integrals for $n=2, \ldots, 6$. Although all approaches have identical basis elements and thus have to have equivalent tensor coefficients when compared after complete reduction, we would like to stress that they allow for quite different algorithmic realizations.

\footnotetext{
1 We observed problems in certain configurations with light-like external particles.
} 
The Letter is organized as follows. Basic formulae are introduced in Section 2. Section 3 contains the main result, the recursive tensor reduction, based on master representations for the $(5, R)$-integrals. As a demonstration, we derive the $(5,4)$-integrals in more detail in Section 4. Section 5 is a short comment on the $(6, R)$-integral reductions. In Section 6 we state some properties of the auxiliary vectors used in the recursions, which also allow for an alternative and simple derivation of our master formula. We finish with a short Section 7.

\section{Basic formulae}

We study Feynman tensor integrals in the generic dimension $d=$ $4-2 \epsilon$ with $n$ external legs:

$I_{n}^{\mu_{1} \cdots \mu_{R}}=\int \frac{d^{d} k}{i \pi^{d / 2}} \frac{\prod_{r=1}^{R} k^{\mu_{r}}}{\prod_{j=1}^{n} c_{j}^{v_{j}}}$,

where the denominators $c_{j}$ have indices $v_{j}$ and chords $q_{j}$ :

$c_{j}=\left(k-q_{j}\right)^{2}-m_{j}^{2}+i \varepsilon$.

We will assume $v_{j}=1$ in the following, but a generalization of the results to arbitrary indices is straightforward.

The iteration of reduction steps will be performed until the level of $(n, 0)$-integrals with $n \leqslant 4$ is reached. In this chain, the following well-known scalar reductions [9] are needed:

$I_{n}=\sum_{s=1}^{n} \frac{\left(\begin{array}{l}0 \\ s\end{array}\right)_{n}}{\left(\begin{array}{l}0 \\ 0\end{array}\right)_{n}} I_{n-1}^{s}, \quad n=5,6$.

The simplest, but typical tensor is the vector integral:

$I_{n}^{\mu}=-\sum_{i=1}^{n} q_{i}^{\mu} I_{n, i}^{[d+]}$.

The integrals $I_{n, i}^{[d+]}$ are scalar $n$-point integrals, obtained from $I_{n}$ by raising the index of line $i$ by one unit ( $v_{i}=2$ then) and replacing the generic dimension by dimension $d+2 .^{2}$ In a next step, we apply the recursion relations derived in $[7,9]$ in order to eliminate the shifts of dimension and indices. The details of the derivations, which are relatively easy for lower rank tensors, get complicated, due to many cancellations, for tensors of higher ranks.

The recursion relation for the vector coefficients in (4) reads for $n \leqslant 5:$

$I_{n, i}^{[d+]}=-\frac{\left(\begin{array}{l}0 \\ i\end{array}\right)_{n}}{()_{n}} I_{n}+\sum_{s=1}^{n} \frac{\left(\begin{array}{l}s \\ i\end{array}\right)_{n}}{()_{n}} I_{n-1}^{s}$.

For $n=1$ the second term in (5) vanishes and for $n>5$ the denominator in (5) is ()$_{n}=0$. The case $n=6$ is of practical importance and will be discussed below. $I_{n}$ is the scalar integral, and $I_{n-1}^{s}$ the integral $I_{n}$ where line $s$ has been scratched. ${ }^{3}$ The objects like $\left(\begin{array}{l}S \\ i\end{array}\right)_{n}$ are signed minors, and ()$_{n}$ is the modified Cayley determinant. For explicit definitions, see [8] or Appendix A of [5]. ${ }^{4}$ Thus, we can write for the vector $n$-point function [i.e. the $(n, 1)$ integral] (4) for $n \leqslant 5$ :

$I_{n}^{\mu}=I_{n} Q_{0}^{\mu}-\sum_{s=1}^{n} I_{n-1}^{s} Q_{s}^{\mu}$.

\footnotetext{
2 Analogously, $I_{n, i j}^{[d+]^{2}}$ has indices $v_{i}=2$ and $v_{j}=2$ and is defined in $d+4$ dimensions, etc.

3 Analogously, in $I_{n-2}^{\{\mu\}, s t}$ lines $s$ and $t$ have been scratched, etc.

4 The Gram determinant is $\left|2 q_{j} q_{k}\right|, j, k=1, \ldots, n-1$, and for $q_{n}=0$ we have ()$_{n}=-\left|2 q_{j} q_{k}\right|$.
}

In (6) we introduced the auxiliary vectors $Q_{s}^{\mu}$ :

$Q_{s}^{\mu}=\sum_{i=1}^{n} q_{i}^{\mu} \frac{\left(\begin{array}{l}s \\ i\end{array}\right)_{n}}{()_{n}}, \quad s=0, \ldots, n$.

Vectors (7) are universal and will appear in more involved reductions again. Indeed, (6) is what we want to obtain further on, i.e. we will look for analogous relations for higher rank tensors in the following.

Eq. (6) is essentially due to recursion relation (30) of [9], which reduces simultaneously dimension and index (let us call it type I recursion). For ( $n, R)$-integrals with rank $R \geqslant 2$, a complication arises due to the appearance of the $g^{\mu \nu}$-tensor in the reduction to scalar functions, as may be seen from the simplest case of an $(n, 2)$ integral:

$I_{n}^{\mu \nu}=\sum_{i, j=1}^{n} q_{i}^{\mu} q_{j}^{\nu} v_{i j} I_{n, i j}^{[d+]^{2}}-\frac{1}{2} g^{\mu v} I_{n}^{[d+]}$,

with $v_{i j}=1+\delta_{i j}$. In $d=4$ dimensions, one may eliminate $g^{\mu \nu}$ by expressing it in terms of the $n$ different chords of the integral:

$g^{\mu \nu}=2 \sum_{i, j=1}^{6} q_{i}^{\mu} q_{j}^{v} \frac{\left(\begin{array}{l}0 i \\ 0 j\end{array}\right)_{6}}{\left(\begin{array}{l}0 \\ 0\end{array}\right)_{6}}$,

$g^{\mu \nu}=2 \sum_{i, j=1}^{5} q_{i}^{\mu} q_{j}^{\nu} \frac{\left(\begin{array}{l}i \\ j\end{array}\right)_{5}}{()_{5}}$,

$g^{\mu \nu}=2 \sum_{i, j=1}^{4} q_{i}^{\mu} q_{j}^{v} \frac{\left(\begin{array}{l}i \\ j\end{array}\right)_{4}}{()_{4}}+\frac{8 v^{\mu} v^{v}}{()_{4}}$,

$g^{\mu \nu}=2 \sum_{i, j=1}^{3} q_{i}^{\mu} q_{j}^{v} \frac{\left(\begin{array}{l}i \\ j\end{array}\right)_{3}}{()_{3}}+\frac{4 v^{\mu \lambda} v_{\lambda}^{v}}{()_{3}}$.

For $n=6,5$, see [9]. For $n<5$, we have to introduce extra terms [24], defined with the aid of:

$v^{\mu}=\varepsilon^{\mu \lambda \rho \sigma}\left(q_{1}-q_{4}\right)_{\lambda}\left(q_{2}-q_{4}\right)_{\rho}\left(q_{3}-q_{4}\right)_{\sigma}$,

$v^{\mu \lambda}=\varepsilon^{\mu \lambda \rho \sigma}\left(q_{1}-q_{3}\right)_{\rho}\left(q_{2}-q_{3}\right)_{\sigma}$,

where

$$
\begin{aligned}
v^{\mu \lambda} v_{\lambda}^{\nu}= & q_{1}^{2} q_{2}^{\mu} q_{2}^{\nu}+q_{2}^{2} q_{1}^{\mu} q_{1}^{\nu}-\left(q_{1} q_{2}\right)\left(q_{1}^{\mu} q_{2}^{\nu}+q_{1}^{\nu} q_{2}^{\mu}\right) \\
& -\left[q_{1}^{2} q_{2}^{2}-\left(q_{1} q_{2}\right)^{2}\right] g^{\mu \nu} .
\end{aligned}
$$

In (15), the $q_{1}, q_{2}$ are short for the two 4-vectors in (14), $\left(q_{i}-q_{3}\right)$; $i=1,2$. We just mention that in our conventions $\operatorname{det}\left(g^{\mu \nu}\right)=-1$ and $\varepsilon^{0123}=-\varepsilon_{0123}=+1$. Further, $v^{2}=\frac{1}{8}()_{4}$. It is also interesting to note that the contractions of the sums appearing in (11) and (12) with their corresponding extra terms vanish, i.e. these terms are orthogonal.

Now, we are ready to derive a systematic recursion algorithm.

\section{The $(5, R)$-integrals}

The scalar $(5,0)$-integral is given in $(3)$, and the vector $(5,1)$ integrals in (6). Applying recursion relations, one may derive the following master formula for the $(5, R)$-integrals:

$I_{5}^{\mu_{1} \ldots \mu_{R-1} \mu}=I_{5}^{\mu_{1} \ldots \mu_{R-1}} Q_{0}^{\mu}-\sum_{s=1}^{5} I_{4}^{\mu_{1} \ldots \mu_{R-1}, s} Q_{s}^{\mu}$.

The formula is a generalization of (6). Eq. (16) is given implicitly in [5], for $n=5, R \leqslant 3$ : by Eq. (3.7) for $R=2$ and by Eq. (3.19) for 
$R=3$, both in combination with Eqs. (2.1) and (2.2) (these both for $n=4$ ). For $R=4$ see also Section 4 and, alternatively, see also Section 6.

As a consequence, (16) has a completely new tensor structure compared to what one is used to. The explicit evaluation of (16) will be discussed below for $R=3,4,5$. The $(5,2)$-integrals follow immediately from (16) since the vector integrals $I_{5}^{\mu}$ and $I_{4}^{\mu, s}$ on the r.h.s. are known from (6).

\subsection{The $(5,3)$-integral recursion family}

The master formula $(16)$ for $(5,3)$-integrals has on the r.h.s. $(5,2)$ - and $(4,2)$-integrals:

$I_{5}^{\mu_{1} \mu_{2} \mu}=I_{5}^{\mu_{1} \mu_{2}} Q_{0}^{\mu}-\sum_{s=1}^{5} I_{4}^{\mu_{1} \mu_{2}, s} Q_{s}^{\mu}$.

The $(5,2)$-integrals have already been discussed and may be expressed by $(5,1)$ - and $(4,1)$-integrals according to (16) and (6). For the $(4,2)$-integrals, we need now a reduction analogous to the master formula (16) and may use as a starting point (8). The dimensional shifts in (8) may be treated with a reduction of type I:

$$
\begin{aligned}
v_{i j} I_{4, i j}^{[d+]^{2}, s}= & -\frac{\left(\begin{array}{c}
0 s \\
j s
\end{array}\right)_{5}}{\left(\begin{array}{l}
s \\
s
\end{array}\right)_{5}} I_{4, i}^{[d+], s}+\sum_{t=1, \neq i}^{5} \frac{\left(\begin{array}{c}
t s \\
j s
\end{array}\right)_{5}}{\left(\begin{array}{l}
s \\
s
\end{array}\right)_{5}} I_{3, i}^{[d+], s t} \\
& +\frac{\left(\begin{array}{l}
i s \\
j s
\end{array}\right)_{5}}{\left(\begin{array}{l}
s \\
s
\end{array}\right)_{5}} I_{4}^{[d+], s},
\end{aligned}
$$

and another one, needed for the reduction of the dimension only (not of an index), let us call it reduction of type II. Eq. (31) of [9] yields:

$I_{4}^{[d+], s}=\frac{\left(\begin{array}{c}0 s \\ 0 s\end{array}\right)_{5}}{\left(\begin{array}{l}s \\ s\end{array}\right)_{5}} I_{4}^{s}-\sum_{t=1}^{5} \frac{\left(\begin{array}{c}t s \\ 0 s\end{array}\right)_{5}}{\left(\begin{array}{l}s \\ s\end{array}\right)_{5}} I_{3}^{s t}$.

After eliminating the $g^{\mu \nu}$ in (8) with the aid of (11), we obtain:

$I_{4}^{\mu \nu, s}=I_{4}^{\mu, s} Q_{0}^{s, \nu}-\sum_{t=1}^{4} I_{3}^{\mu, s t} Q_{t}^{s, \nu}-\frac{4 v^{s, \mu} v^{s, v}}{()_{4}} I_{4}^{[d+], s}$.

The notations $Q_{t}^{s, v}$ and $v^{s, v}$ mean that from the five chords of the 5 -point function the chord $s$ is excluded, such that these vectors are constructed from four chords-as given in (11). In fact, (20) together with (19) is the reduction of the $(4,2)$-integrals, when it is combined with (6) and:

$$
\begin{aligned}
I_{3}^{\mu, s t} & =I_{3}^{s t} \sum_{i=1}^{5} q_{i}^{\mu} \frac{\left(\begin{array}{c}
0 s t \\
i s t
\end{array}\right)_{5}}{\left(\begin{array}{c}
s t \\
s t
\end{array}\right)_{5}}-\sum_{u=1}^{5} I_{2}^{s t u} \sum_{i=1}^{5} q_{i}^{\mu} \frac{\left(\begin{array}{c}
u s t \\
i s t
\end{array}\right)_{5}}{\left(\begin{array}{c}
s t \\
s t
\end{array}\right)_{5}} \\
& \equiv I_{3}^{s t} Q_{0}^{s t, \mu}-\sum_{u=1}^{5} I_{2}^{u s t} Q_{u}^{s t, \mu},
\end{aligned}
$$

where the upper indices $s, t$ in the $Q^{s t}$-vectors are again introduced for the scratched lines. Observe that for $i=s, t$ and $u=s, t$ there are no contributions so that indeed the indices are running only over three values and objects like $\left(\begin{array}{l}0 s t \\ i s t\end{array}\right)_{5}$ can indeed be read as $\left(\begin{array}{l}0 \\ i\end{array}\right)_{3}$. In this way (21) is consistent with (6). If one is interested in 4-point functions from the very beginning one avoids of course this clumsy notation-but for the present purpose of reducing 5point tensors to scalars it appears adequate to demonstrate this at least once.

This completes the $(5,3)-,(4,2)-$, and $(3,1)$-integral recursions.

\subsection{The $(5,4)$-integral recursion family}

For the higher tensors of the 5-point function we need correspondingly higher tensors of the 4-point functions, and the corresponding extra terms related to the elimination of the $g^{\mu \nu}$ have to be derived.

Thus, as a next step we seek a representation for the $(4,3)$ integrals which is needed for the $(5,4)$-integral recursion:

$I_{5}^{\mu_{1} \mu_{2} \mu_{3} \mu}=I_{5}^{\mu_{1} \mu_{2} \mu_{3}} Q_{0}^{\mu}-\sum_{s=1}^{5} I_{4}^{\mu_{1} \mu_{2} \mu_{3}, s} Q_{s}^{\mu}$,

but is also of interest in its own. In the following, for the ease of notation, we will drop the scratches of line $s$.

A systematic application of recursions of type I results in:

$$
\begin{aligned}
I_{4}^{\mu \nu \lambda}= & I_{4}^{\mu \nu} Q_{0}^{\lambda}-\sum_{t=1}^{4} I_{3}^{\mu \nu, t} Q_{t}^{\lambda}-G^{\mu \lambda} I_{4}^{\nu,[d+]} \\
& -G^{\nu \lambda} I_{4}^{\mu,[d+]},
\end{aligned}
$$

with (see also (11)):

$G^{\mu \lambda}=\frac{1}{2} g^{\mu \lambda}-\sum_{i, j=1}^{4} q_{i}^{\mu} q_{i}^{\lambda} \frac{\left(\begin{array}{l}i \\ j\end{array}\right)_{4}}{()_{4}}=\frac{4 v^{\mu} v^{\nu}}{()_{4}}$,

and

$I_{4}^{\mu,[d+]}=-\sum_{k=1}^{4} q_{k}^{\mu} I_{4, k}^{[d+]^{2}}=I_{4}^{[d+]} Q_{0}^{\mu}-\sum_{t=1}^{4} I_{3}^{[d+], t} Q_{t}^{\mu}$.

In $I_{4}^{\mu,[d+]}$, besides $I_{4}^{[d+]}$ (known from (19)), also $I_{3}^{[d+], t}$ enters. It may be reduced by a recursion of type II quite similar to (19):

$I_{3}^{[d+], t}=\left[\frac{\left(\begin{array}{l}0 t \\ 0 t\end{array}\right)_{4}}{\left(\begin{array}{l}t \\ t\end{array}\right)_{4}} I_{3}^{t}-\sum_{u=1}^{4} \frac{\left(\begin{array}{l}u t \\ 0 t\end{array}\right)_{4}}{\left(\begin{array}{l}t \\ t\end{array}\right)_{4}} I_{2}^{t u}\right] \frac{1}{d-2}$.

Finally, our representation (23) of $I_{4}^{\mu \nu \lambda}$ contains the integrals $I_{3}^{\mu \nu, t}$ and we have to reduce them also. Application of recursion relations yields the analogue of (20) for $n=4$ :

$I_{3}^{\mu \nu, t}=I_{3}^{\mu, t} Q_{0}^{t, v}-\sum_{u=1}^{4} I_{2}^{\mu, t u} Q_{u}^{t, v}-I_{3}^{[d+], t} \frac{2 v^{t, \mu \lambda} v_{\lambda}^{t, v}}{\left(\begin{array}{l}t \\ t\end{array}\right)_{4}}$.

We made use of the definition (7), which becomes here:

$Q_{u}^{t, v}=\sum_{i=1}^{4} q_{i}^{v} \frac{\left(\begin{array}{c}u t \\ i t\end{array}\right)_{4}}{\left(\begin{array}{l}t \\ t\end{array}\right)_{4}}, \quad u=0, \ldots, 4$,

and of the representation (12) in order to express:

$G^{t, \mu \nu}=\frac{1}{2} g^{\mu \nu}-\sum_{i, j=1}^{4} q_{i}^{\mu} q_{j}^{\nu} \frac{\left(\begin{array}{l}i t \\ j t\end{array}\right)_{4}}{\left(\begin{array}{l}t \\ t\end{array}\right)_{4}}=\frac{2 v^{t, \mu \lambda} v_{\lambda}^{t, v}}{\left(\begin{array}{l}t \\ t\end{array}\right)_{4}}$.

This completes the $(5,4)-,(4,3)-$, and $(3,2)$-integral recursions.

\subsection{The $(5,5)$-integral recursion family}

For the tensor of rank 5 of the 5-point function we need further the tensor of rank 4 of the 4 -point function,

$I_{5}^{\mu_{1} \mu_{2} \mu_{3} \mu_{4} \mu}=I_{5}^{\mu_{1} \mu_{2} \mu_{3} \mu_{4}} Q_{0}^{\mu}-\sum_{s=1}^{5} I_{4}^{\mu_{1} \mu_{2} \mu_{3} \mu_{4}, s} Q_{s}^{\mu}$. 
Again, the systematic application of the recursion relations results in

$$
\begin{aligned}
I_{4}^{\mu \nu \lambda \rho}= & I_{4}^{\mu \nu \lambda} Q_{0}^{\rho}-\sum_{t=1}^{4} I_{3}^{\mu \nu \lambda, t} Q_{t}^{\rho} \\
& -G^{\mu \rho} T^{\nu \lambda}-G^{\nu \rho} T^{\mu \lambda}-G^{\lambda \rho} T^{\mu \nu}
\end{aligned}
$$

with

$T^{\mu \nu}=I_{4}^{\mu,[d+]} Q_{0}^{v}-\sum_{t=1}^{4} I_{3}^{\mu,[d+], t} Q_{t}^{v}-G^{\mu \nu} I_{4}^{[d+]^{2}}$,

where $G^{\mu \nu}$ and $I_{4}^{\mu,[d+]}$ are given in (24) and (25), respectively, and

$$
\begin{aligned}
I_{3}^{\mu,[d+], t} & =-\sum_{i=1}^{4} q_{i}^{\mu} I_{3, i}^{[d+]^{2}, t} \\
& =I_{3}^{[d+], t} Q_{0}^{t, \mu}-\sum_{u=1}^{4} I_{2}^{[d+], t u} Q_{u}^{t, \mu} .
\end{aligned}
$$

The $I_{3}^{[d+], t}$ is known from (26), the $I_{4}^{[d+]}$ from (19), and for completeness we specify also the other recursions of type II:

$$
\begin{aligned}
& I_{4}^{[d+]^{2}}=\left[\frac{\left(\begin{array}{l}
0 \\
0
\end{array}\right)_{4}}{()_{4}} I_{4}^{[d+]}-\sum_{t=1}^{4} \frac{\left(\begin{array}{l}
t \\
0
\end{array}\right)_{4}}{()_{4}} I_{3}^{[d+], t}\right] \frac{1}{d-1}, \\
& I_{2}^{[d+], t u}=\left[\frac{\left(\begin{array}{c}
0 t u \\
0 t u
\end{array}\right)_{4}}{\left(\begin{array}{c}
t u \\
t u
\end{array}\right)_{4}} I_{2}^{t u}-\sum_{v=1}^{4} \frac{\left(\begin{array}{l}
0 t u \\
v t u
\end{array}\right)_{4}}{\left(\begin{array}{l}
t u \\
t u
\end{array}\right)_{4}} I_{1}^{t u v}\right] \frac{1}{d-1} .
\end{aligned}
$$

In (31) enters also the $I_{3}^{\mu \nu \lambda, t}$, which we evaluate to be:

$$
\begin{aligned}
I_{3}^{\mu \nu \lambda, t}= & I_{3}^{\mu \nu, t} Q_{0}^{t, \lambda}-\sum_{u=1}^{4} I_{2}^{\mu \nu, t u} Q_{u}^{t, \lambda} \\
& -G^{t, \mu \lambda} I_{3}^{\nu,[d+], t}-G^{t, \nu \lambda} I_{3}^{\mu,[d+], t},
\end{aligned}
$$

where $G^{t, \mu v}$ is given in (29) and $I_{3}^{\mu,[d+], t}$ in (33).

It remains to evaluate the tensor $I_{2}^{\mu \nu, t u}$, for which we get:

$I_{2}^{\mu \nu, t u}=I_{2}^{\mu, t u} Q_{0}^{t u, v}-\sum_{v=1}^{4} I_{1}^{\mu, t u v} Q_{v}^{t u, v}-G^{t u, \mu v} I_{2}^{[d+], t u}$.

The auxiliary vector $Q_{v}^{t u, v}$, defined analogously to (28), vanishes when $v=t$ or $v=u$. The sum over $v$ in (37) therefore consists of two terms, $v=i, i^{\prime}$, where $i<i^{\prime}$ and $i, i^{\prime} \neq t, u$. With $\left(\begin{array}{c}t u \\ t u\end{array}\right)_{4}=$ $-2\left(q_{i}-q_{i^{\prime}}\right)^{2} \equiv-2 q^{2}$ we have for the vector basis in (37):

$Q_{0}^{t u, v}=\frac{1}{2}\left(q_{i}+q_{i^{\prime}}\right)^{v}-\frac{1}{2 q^{2}}\left(m_{i}^{2}-m_{i^{\prime}}^{2}\right)\left(q_{i}-q_{i^{\prime}}\right)^{v}$,

$Q_{i}^{t u, v}=-Q_{i^{\prime}}^{t u, v}=\frac{1}{2 q^{2}}\left(q_{i}-q_{i^{\prime}}\right)^{v}$,

$G^{t u, \mu \nu}=\frac{1}{2}\left(g^{\mu \nu}-\frac{\left(q_{i}-q_{i^{\prime}}\right)^{\mu}\left(q_{i}-q_{i^{\prime}}\right)^{v}}{q^{2}}\right)$.

Finally, in (37) appear the integrals:

$$
\begin{aligned}
I_{2}^{\mu, t u}= & I_{2}^{t u} Q_{0}^{t u, \mu}-\sum_{v=1}^{4} I_{1}^{t u v} Q_{v}^{t u, \mu} \\
= & \frac{1}{2} I_{2}^{t u}\left(q_{i}+q_{i^{\prime}}\right)^{\mu} \\
& -\frac{1}{2}\left(m_{i}^{2}-m_{i^{\prime}}^{2}\right) \frac{I_{2}^{t u}\left(q^{2}\right)-I_{2}^{t u}(0)}{q^{2}}\left(q_{i}-q_{i^{\prime}}\right)^{\mu}
\end{aligned}
$$

and

$I_{1}^{\mu, t u v}=-q_{i}^{\mu} I_{1, i}^{[d+], t u v}=q_{i} I_{1}^{t u v}, \quad i \neq t, u, v$.

The last equality in (42) follows from:

$I_{1, i}^{[d+], t u v}=-\frac{\left(\begin{array}{c}0 t u v \\ i t u v\end{array}\right)_{4}}{\left(\begin{array}{c}t u v \\ \text { tuv }\end{array}\right)_{4}} I_{1}^{\text {tuv }}=-I_{1}^{\text {tuv }}$.

This completes the $(5,5)-,(4,4)-,(3,3)-,(2,2)-$ and $(1,1)-$ integral recursions.

\section{Derivation of the master formula for $(5,4)$-integrals}

As mentioned before, (16) is implicitly contained in [5], but only up to $R=3$. We will make use of it, however, up to $R=5$ at least. To demonstrate the general approach of how to obtain this relation, we give details of its analytic proof for $R=4$. In particular we observe huge cancellations of higher dimensional integrals in (2.4) of [5]. To start with, as usual we write this relation as:

$I_{5}^{\mu \nu \lambda \rho}=\sum_{i, j, k, l=1}^{5} q_{i}^{\mu} q_{j}^{\nu} q_{k}^{\lambda} q_{l}^{\rho} I_{5, i j k l}$,

where (10) has to be used. The highest dimensional integral occurring now is $n_{i j k l} I_{5, i j k l}^{[d+]^{4}}$, with $n_{i j k l}=v_{i j} v_{i j k} v_{i j k l}, v_{i j k l}=1+\delta_{i l}+\delta_{j l}+\delta_{k l}$, etc., for which we need the recursion relation:

$$
\begin{aligned}
v_{i j k l} I_{5, i j k l}^{[d+]^{4}}= & -\frac{\left(\begin{array}{l}
0 \\
l
\end{array}\right)_{5}}{()_{5}} I_{5, i j k}^{[d+]^{3}}+\sum_{\substack{s=1 \\
s \neq i, j, k}}^{5} \frac{\left(\begin{array}{l}
s \\
l
\end{array}\right)_{5}}{()_{5}} I_{4, i j k}^{[d+]^{3}, s} \\
& +\frac{\left(\begin{array}{l}
i \\
l
\end{array}\right)_{5}}{()_{5}} I_{5, j k}^{[d+]^{3}}+\frac{\left(\begin{array}{l}
j \\
l
\end{array}\right)_{5}}{()_{5}} I_{5, i k}^{[d+]^{3}}+\frac{\left(\begin{array}{l}
k \\
l
\end{array}\right)_{5}}{()_{5}} I_{5, i j}^{[d+]^{3}} .
\end{aligned}
$$

In the sum of the last three terms of (45), to be abbreviated as $[i j k]^{(l)}$, it is understood that there occur no equal indices among $i, j, k$ (otherwise it would be contained as a 4-point function in the second term on the right-hand side of (45)). With the remaining factor $v_{i j} v_{i j k}$ of the integral $n_{i j k l} I_{5, i j k l}^{[d+]^{4}}$ we can rewrite:

$$
\begin{aligned}
v_{i j} v_{i j k}[i j k]^{(l)}= & v_{j k} \frac{\left(\begin{array}{l}
i \\
l
\end{array}\right)_{5}}{()_{5}} I_{5, j k}^{[d+]^{3}}+v_{i k} \frac{\left(\begin{array}{l}
j \\
l
\end{array}\right)_{5}}{()_{5}} I_{5, i k}^{[d+]^{3}} \\
& +v_{i j} \frac{\left(\begin{array}{l}
k \\
l
\end{array}\right)_{5}}{()_{5}} I_{5, i j}^{[d+]^{3}},
\end{aligned}
$$

and as a result we have:

$$
\begin{aligned}
& I_{5, i j k l}=v_{i j} v_{i j k}\left[-\frac{\left(\begin{array}{l}
0 \\
l
\end{array}\right)_{5}}{()_{5}} I_{5, i j k}^{[d+]^{3}}+\sum_{s=1, s \neq i, j, k}^{5} \frac{\left(\begin{array}{l}
s \\
l
\end{array}\right)_{5}}{()_{5}} I_{4, i j k}^{[d+]^{3}, s}\right] \\
& -\left[v_{k l} \frac{\left(\begin{array}{l}
i \\
j
\end{array}\right)_{5}}{()_{5}} I_{5, k l}^{[d+]^{3}}+v_{j l} \frac{\left(\begin{array}{l}
i \\
k
\end{array}\right)_{5}}{()_{5}} I_{5, j l}^{[d+]^{3}}+v_{i l} \frac{\left(\begin{array}{l}
j \\
k
\end{array}\right)_{5}}{()_{5}} I_{5, i l}^{[d+]^{3}}\right] \\
& +\frac{1}{()_{5}^{2}}\left[\left(\begin{array}{l}
i \\
j
\end{array}\right)_{5}\left(\begin{array}{l}
k \\
l
\end{array}\right)_{5}+\left(\begin{array}{l}
i \\
k
\end{array}\right)_{5}\left(\begin{array}{l}
j \\
l
\end{array}\right)_{5}\right. \\
& \left.+\left(\begin{array}{l}
j \\
k
\end{array}\right)_{5}\left(\begin{array}{l}
i \\
l
\end{array}\right)_{5}\right] I_{5}^{[d+]^{2}},
\end{aligned}
$$

where (46) has already completely cancelled against the last three terms of the second sum of (2.4) in [5]. With the further recursion: 


$$
\begin{aligned}
v_{i l} I_{5, i l}^{[d+]^{3}}= & -\frac{\left(\begin{array}{l}
0 \\
l
\end{array}\right)_{5}}{()_{5}} I_{5, i}^{[d+]^{2}}+\sum_{\substack{s=1 \\
s \neq i}}^{5} \frac{\left(\begin{array}{l}
s \\
l
\end{array}\right)_{5}}{()_{5}} I_{4, i}^{[d+]^{2}, s} \\
& +\frac{\left(\begin{array}{l}
i \\
l
\end{array}\right)_{5}}{()_{5}} I_{5}^{[d+]^{2}},
\end{aligned}
$$

one observes that the $I_{5}^{[d+]^{2}}$ term cancels against the last row of (47) and the remaining $I_{5}$ integrals combine to $I_{5, i j k}$ according to (3.18) of [5] with the result:

$$
\begin{aligned}
I_{5, i j k l}= & \frac{\left(\begin{array}{l}
0 \\
l
\end{array}\right)_{5}}{()_{5}} I_{5, i j k} \\
& -\left[\frac{\left(\begin{array}{l}
i \\
j
\end{array}\right)_{5}}{()_{5}} \sum_{\substack{s=1 \\
s \neq k}}^{5} \frac{\left(\begin{array}{l}
s \\
l
\end{array}\right)_{5}}{()_{5}} I_{4, k}^{[d+]^{2}, s}+(i \leftrightarrow k)+(j \leftrightarrow k)\right] \\
& +v_{i j} v_{i j k} \sum_{\substack{s=1 \\
s \neq i, j, k}}^{5} \frac{\left(\begin{array}{l}
s \\
l
\end{array}\right)_{5}}{()_{5}} I_{4, i j k}^{[d+]^{3}, s} .
\end{aligned}
$$

This proves our statement: The first term corresponds to the $(5,3)$ integral as claimed in (16). The rest, according to (2.3) of [5], corresponds to the $(4,3)$-integral, again corresponding to (16). A similar proof for $n=5, R=5$, Eq. (30), is a bit more lengthy, but see also the derivation in Section 6. We would like to mention that a pedagogical introduction to the techniques applied may be found in [25].

\section{The $(6, R)$-integrals}

Representing the $g^{\mu \nu}$-tensor by (9), one has the analogue of (16) for the $(6, R)$-integrals:

$$
I_{6}^{\mu_{1} \ldots \mu_{R-1} \rho}=-\sum_{s=1}^{6} I_{5}^{\mu_{1} \ldots \mu_{R-1}, s} \bar{Q}_{s}^{\rho},
$$

where the auxiliary vectors $\bar{Q}_{s}$ read:

$$
\bar{Q}_{s}^{\rho}=\sum_{i=1}^{6} q_{i}^{\rho} \frac{\left(\begin{array}{c}
0 s \\
0 i
\end{array}\right)_{6}}{\left(\begin{array}{l}
0 \\
0
\end{array}\right)_{6}}, \quad s=1, \ldots, 6 .
$$

Since $\left(\begin{array}{l}00 \\ 0 i\end{array}\right)_{6}=0$, the analogue of the first term in (16) with vector $\bar{Q}_{0}^{\rho}=\sum_{i=1}^{6} q_{i}^{\mu}\left(\begin{array}{c}00 \\ 0 i\end{array}\right)_{6} /\left(\begin{array}{l}0 \\ 0\end{array}\right)_{6}$ vanishes. Eq. (50) is well known $[5,9,14$, $16]$. We only mention it in order to show how nicely it fits into our general scheme.

\section{Discussion of the auxiliary vectors $Q_{s}^{\mu}$}

Finally we want to investigate properties of the auxiliary vectors $Q_{0}^{\mu}, Q_{s}^{\mu}$ defined in (7). Of particular practical interest is the contraction of a tensor integral with a chord. ${ }^{5}$ Usually, a scalar product $q_{i} k$ is expressed in terms of the difference of two propagators, which can then be cancelled such that a tensor integral of lower degree is obtained. This simplification is often the first step considering the original diagram, where one may further select $q_{n}=0$. Our approach offers an alternative due to the fact that the contraction of one of the vectors (7) with a chord yields a simple expression (utilizing here the choice $q_{n}=0$ ):

5 Remember that every external momentum may be expressed by the chords.

$$
\begin{gathered}
q_{i} Q_{0}=\sum_{j=1}^{n-1} q_{i} q_{j} \frac{\left(\begin{array}{l}
0 \\
j
\end{array}\right)_{n}}{()_{n}}=-\frac{1}{2}\left(Y_{i n}-Y_{n n}\right), \\
i=1, \ldots, n-1, \\
q_{i} Q_{s}=\sum_{j=1}^{n-1} q_{i} q_{j} \frac{\left(\begin{array}{l}
s \\
j
\end{array}\right)_{n}}{()_{n}}=\frac{1}{2}\left(\delta_{i s}-\delta_{n s}\right), \\
i=1, \ldots, n-1, s=1, \ldots, n,
\end{gathered}
$$

with

$Y_{j k}=-\left(q_{j}-q_{k}\right)^{2}+m_{j}^{2}+m_{k}^{2}$.

Further we observe that the contraction of any of the extra terms introduced in (11) and (12) with a chord vanishes (for $q_{n}=0$ ). In this way, we have not only reduced the rank of the tensor by one, but we additionally obtain a simple expression in terms of the lower rank tensors.

Relations (52) and (53) also allow a very simple derivation of (16) for $q_{5}=0$ by projecting with a complete set of chords. Writing (16) as:

$$
\begin{aligned}
\int \frac{k^{\mu_{1}} \cdots k^{\mu_{R-1}}\left(k q_{k}\right)}{c_{1} c_{2} c_{3} c_{4} c_{5}}= & \int \frac{k^{\mu_{1}} \cdots k^{\mu_{R-1}}}{c_{1} c_{2} c_{3} c_{4} c_{5}}\left(Q_{0} q_{k}\right) \\
& -\sum_{s=1}^{5} \int \frac{k^{\mu_{1}} k^{\mu_{R-1}} \cdot c_{s}}{c_{1} c_{2} c_{3} c_{4} c_{5}}\left(Q_{s} q_{k}\right),
\end{aligned}
$$

with $k q_{k}=-\frac{1}{2}\left[c_{k}-c_{5}+Y_{k 5}-Y_{55}\right]$, from (52) and (53) immediately follows the equality.

For $q_{5} \neq 0$ a shift, $k \rightarrow k+q_{5}$, is needed on the integration momentum $k$ of the original integral. Under such a shift all vectors $q_{i}$ are shifted to $q_{i} \rightarrow q_{i}-q_{5}$. The $Q_{s}$ for $s=1, \ldots, 5$ stay invariant, and the $Q_{0}$ shifts like a chord:

$$
\begin{aligned}
Q_{0} & =\sum_{j=1}^{5} q_{j} \frac{\left(\begin{array}{l}
0 \\
j
\end{array}\right)_{5}}{()_{5}} \rightarrow \sum_{j=1}^{5}\left(q_{j}-q_{5}\right) \frac{\left(\begin{array}{l}
0 \\
j
\end{array}\right)_{5}}{()_{5}} \\
& =Q_{0}-q_{5} .
\end{aligned}
$$

This is due to the fact that the Gram determinants are invariant under any shift and

$$
\begin{aligned}
& \sum_{j=1}^{5}\left(\begin{array}{l}
0 \\
j
\end{array}\right)_{5}=()_{5}, \\
& \sum_{j=1}^{5}\left(\begin{array}{l}
s \\
j
\end{array}\right)_{5}=0, \quad s=1, \ldots, 5 .
\end{aligned}
$$

After the shift one has only integrals with $q_{5}=0$. Collecting all contributions and shifting back, one obtains (16).

The auxiliary vectors $Q_{s}$ introduce in the recursions inverse powers of ()$_{n}$. Relations (52) and (53) yield:

$Q_{0}^{2}=\frac{1}{2} \frac{\left(\begin{array}{l}0 \\ 0\end{array}\right)_{n}}{()_{n}}+\frac{1}{2} Y_{n n}$,

$Q_{s}^{2}=\frac{1}{2} \frac{\left(\begin{array}{l}s \\ s\end{array}\right)_{n}}{()_{n}}, \quad s=1, \ldots, n$.

Eqs. (59) and (60) might suggest that after contractions with chords the effective inverse powers of ()$_{n}$ are reduced. This is not true in general. For $n=5$, however, this can be shown as follows: We write (52) as a system of linear equations for the vector $Q_{0}^{\mu}$ :

$A Q_{0}=x$, 
with

$A=\left(q_{i \mu}\right)$,

$x=\left(\begin{array}{c}x_{1} \\ \cdots \\ x_{4}\end{array}\right)$,

$x_{i}=-\frac{1}{2}\left(Y_{i 5}-Y_{55}\right)$.

Obviously, the matrix $A$ satisfies

$A_{i \mu} g^{\mu v} A_{j v}=q_{i} q_{j}, \quad i, j=1, \ldots 4$,

and hence,

$\operatorname{det}\left(q_{i} q_{j}\right)=-\frac{1}{2^{4}}()_{5}=-\operatorname{det}^{2}(A)$.

Therefore,

$A^{-1} \sim()_{5}^{-\frac{1}{2}}$.

Solving the system (61) yields

$Q_{0}=A^{-1} x \sim() 5^{-\frac{1}{2}}$.

Thus the vector $Q_{0}$ is proportional to ()$_{5}^{-1 / 2}$, while its additive components in (7) are proportional to ()$_{5}^{-1}$. The same obviously applies for the auxiliary vectors $Q_{s}(s=1, \ldots, 5)$.

\section{Summary}

We have presented a new, recursive reduction scheme for oneloop $n$-point tensor Feynman integrals, and derived explicit expressions covering tensors up to rank $R=n$, with $n \leqslant 6$. The crucial point is the derivation of relation (16), which expresses $(5, R)$ integrals in terms of $(5, R-1)$ - and $(4, R-1)$-integrals, and of relations like (31), which express $(k, R)$-integrals $(k \leqslant 4)$ in terms of $(k, R-1)$ - and $(k-1, R-1)$-integrals plus additional terms. Both types of representations make use of auxiliary vectors $Q_{s}^{\mu}$.

The recursive scheme is very convenient for explicit calculations and contains the complete calculational chain of tensor reduction, for both massive and massless propagators, and works with dimensional regularization. The only necessary package to be added is one for the evaluation of 1-point to 4-point scalar integrals. For this we chose the library of scalar functions QCDloop/FF [12, 26] and made careful numerical checks of the recursive reductions. The numerical output was compared, whenever possible, to another, independent Fortran program $[5,10]$. Additionally, 6-point functions have been compared with a program of P. Uwer for massive particles [27], and 5- and 6-point functions with the Golem package [13], which applies for massless particles only. The highest rank covered was $R=5$. We have agreement in all cases, both for the divergent and finite parts of the tensor integrals. Various tables with numerical comparisons may be found in [28].

Differing from the reductions derived in $[5,10]$, one cannot avoid the appearance of inverse Gram determinants ( $)_{5}$ in the recursions. There is a lengthy discussion in the literature, and the rising numerical experience with explicit calculations of cross- sections leads to the conclusion that it is a small fraction of phasespace points which is substantially concerned. For those points, we have to switch to our code based on [5,10], which is more appropriate to the specific kinematics around ()$_{5}=0$. But doing so, the recursive character of the scheme is given up. It is also possible, in the recursion described here, to re-introduce and/or keep integrals in higher dimension in order to avoid inverse Gram determinants, as exemplified in another approach [14].

\section{Acknowledgements}

Work supported in part by Sonderforschungsbereich/Transregio SFB/TRR 9 of DFG "Computergestützte Theoretische Teilchenphysik" and by the European Community's Marie-Curie Research Training Network MRTN-CT-2006-035505 "HEP-TOOLS". J.F. likes to thank DESY for kind hospitality.

\section{References}

[1] G. Passarino, M.J.G. Veltman, Nucl. Phys. B 160 (1979) 151, doi:10.1016/05503213(79)90234-7.

[2] S. Weinzierl, PoS ACAT (2007) 005, arXiv:0707.3342.

[3] Z. Bern, et al., NLO Multileg Working Group, The NLO multileg working group: Summary report, arXiv:hep-ph/0803.0494.

[4] T. Binoth, PoS ACAT (2008) 011, arXiv:0903.1876.

[5] T. Diakonidis, J. Fleischer, J. Gluza, K. Kajda, T. Riemann, J.B. Tausk, Phys. Rev. D 80 (2009) 036003, arXiv:0812.2134.

[6] A.I. Davydychev, Phys. Lett. B 263 (1991) 107, doi:10.1016/03702693(91)91715-8.

[7] O.V. Tarasov, Phys. Rev. D 54 (1996) 6479, arXiv:hep-th/9606018.

[8] D.B. Melrose, Nuovo Cimento 40 (1965) 181.

[9] J. Fleischer, F. Jegerlehner, O. Tarasov, Nucl. Phys. B 566 (2000) 423, arXiv:hepph/9907327.

[10] T. Diakonidis, J. Fleischer, J. Gluza, K. Kajda, T. Riemann, J.B. Tausk, Nucl. Phys. B (Proc. Suppl.) 183 (2008) 109, arXiv:0807.2984.

[11] T. Hahn, M. Perez-Victoria, Comput. Phys. Commun. 118 (1999) 153, arXiv:hep$\mathrm{ph} / 9807565$

[12] G.J. van Oldenborgh, Comput. Phys. Commun. 66 (1991) 1, doi:10.1016/0010 4655(91)90002-3.

[13] T. Binoth, J.P. Guillet, G. Heinrich, E. Pilon, T. Reiter, Comput. Phys. Commun. 180 (2009) 2317, arXiv:0810.0992.

[14] T. Binoth, J. Guillet, G. Heinrich, E. Pilon, C. Schubert, JHEP 0510 (2005) 015 arXiv:hep-ph/0504267.

[15] A. Denner, S. Dittmaier, Nucl. Phys. B 658 (2003) 175, arXiv:hep-ph/0212259.

[16] A. Denner, S. Dittmaier, Nucl. Phys. B 734 (2006) 62, arXiv:hep-ph/0509141.

[17] F. del Aguila, R. Pittau, JHEP 0407 (2004) 017, arXiv:hep-ph/0404120.

[18] A. van Hameren, JHEP 0907 (2009) 088, arXiv:hep-ph/0905.1005.

[19] W.L. van Neerven, J.A.M. Vermaseren, Phys. Lett. B 137 (1984) 241, doi:10.1016/0370-2693(84)90237-5.

[20] Z. Bern, L.J. Dixon, D.A. Kosower, Phys. Lett. B 302 (1993) 299, arXiv:hepph/9212308;

Z. Bern, L.J. Dixon, D.A. Kosower, Phys. Lett. B 318 (1993) 649, Erratum.

[21] Z. Bern, L.J. Dixon, D.A. Kosower, Nucl. Phys. B 412 (1994) 751, arXiv:hep$\mathrm{ph} / 9306240$.

[22] T. Binoth, J.P. Guillet, G. Heinrich, Nucl. Phys. B 572 (2000) 361, arXiv:hep$\mathrm{ph} / 9911342$.

[23] G. Duplancic, B. Nizic, Eur. Phys. J. C 35 (2004) 105, arXiv:hep-ph/0303184.

[24] G.J. van Oldenborgh, J.A.M. Vermaseren, Z. Phys. C 46 (1990) 425, doi:10.1007/BF01621031.

[25] J. Fleischer, Talk at CALC 2009, July 2009, Dubna, Russia, Transparencies at http://theor.jinr.ru/ calc2009/program.html.

[26] R.K. Ellis, G. Zanderighi, JHEP 0802 (2008) 002, arXiv:0712.1851.

[27] P. Uwer, private communication.

[28] T. Diakonidis, Talk at CALC 2009, July 2009, Dubna, Russia, Transparencies at http://theor.jinr.ru/ calc2009/program.html. 